\title{
Validation of a CT-free navigation system for the measurement of native acetabular alignment
}

Shanika MA Arachchi*

Department of Biomedical Engineering, University of Strathclyde, Glasgow UK shanikamihirani@ymail.com

Angelica S. Augustine

Department of Biomedical Engineering, University of Strathclyde, Glasgow UK Department of Orthopaedics, Golden Jubilee National Hospital, Clyde Bank, UK angieshibana@doctors.org.uk

Angela H Deakin

Department of Biomedical Engineering, University of Strathclyde, Glasgow UK Department of Orthopaedics, Golden Jubilee National Hospital, Clyde Bank, UK Angela.Deakin@gjnh.scot.nhs.uk

Frédéric Picard

Department of Biomedical Engineering, University of Strathclyde, Glasgow UK Department of Orthopaedics, Golden Jubilee National Hospital, Clyde Bank, UK frederic.picard@gjnh.scot.nhs.uk

Philp J Rowe

Department of Biomedical Engineering, University of Strathclyde, Glasgow UK philip.rowe@strath.ac.uk

\begin{abstract}
Computer assisted surgery is becoming more frequently used in medical world. OrthoPilot ${ }^{\mathrm{TM}}$ Hip Suite CT-free navigation system (BBraun Aesculap) is one such computer assisted navigation system used for total hip replacement surgery. The validity of OrthoPilot system remains to be determined independent of the manufacturer. The main aims of this study were to investigate the accuracy of OrthoPilot data, while using surgical instruments and to confirm the suitability of the cup navigation algorithm. OrthoPilot was compared with the gold standard of a VICON movement analysis system. An aluminium pelvic phantom was machined with high accuracy to perform the experiments. Data were captured simultaneously from both OrthoPilot and VICON systems and acetabular angles were compared. Both systems produce comparable results for the distance between anatomical landmarks and acetabular angles. It can be concluded that data from OrthoPilot system, if used correctly, are sufficiently accurate for orthopaedic applications.
\end{abstract}

\section{Keywords}

Cup navigation, validation, anteversion angle, inclination angle, Anterior Pelvic Plane, surgical systems, computer assisted surgery, hip prosthesis 


\section{Introduction}

In the UK, approximately $15 \%$ of the female and $10 \%$ of the male population over the age of 65 have radiographic evidence of moderate to severe osteoarthritis of the hip joint [1]. Dandy and Edwards described in their studies that the most common diseases leading to hip replacement are Osteoarthritis, Rheumatoid arthritis, Osteonecrosis and Hip disorders or fractures [2]. According to Frankel et al., 13-18 people per 1000 aged 35-85 years suffer from hip diseases which require surgery [3]. Mal-positioning of the implant is linked with dislocations, impingement, pelvic osteolysis, acetabular migration, leg length discrepancy, early implant wear and malfunction of the hip due to loosening [4]. According to Numair et al., 15\% of revision rate of the acetabular component can be observed in the group of high hip dislocation. This observation was made based on his study at Wrightington hospital, Wigan, UK. Furthermore, cup loosening seems to be a general complication of THA in high hip dislocation and possible loosening of the acetabular component was 16\% [5]. According to Fender et al., the main reasons for implant failure were aseptic loosening (2.3\%), deep infection (1.4\%) and general loosening (5.2\%) [6]. A Swedish study had found $71 \%$ of hip replacement revisions were due to aseptic loosening; however, the number of revisions in Sweden had decreased to 3\% within 10 years mainly due to improvements of surgical techniques [7]. Improper placement of acetabulum or femoral head or both of them may lead to one or a combination of the above-mentioned problems [8].

Navigational techniques have been used in hip replacement surgery for a number of years $[9,10 \& 11]$. Computer assisted navigation has the ability to measure the implant alignment precisely during arthroplasty. The preparation of the acetabular surface and femur thought to be more accurate and precise when navigation is used and resulting in optimal acetabular cup alignment of $45^{\circ}$ of inclination and $20^{\circ}$ anteversion [12]. Computer assisted surgery systems claim to provide optimal implant positioning and minimize the risk of dislocation, impingement and implant wear; hence increasing longevity [11]. In image-free navigation, implant alignment is based only on anatomical landmarks palpated intraoperatively by the surgeon using a reference pointer [13]. This is also called the landmark based navigation.

OrthoPilot $^{\mathrm{TM}}$ Hip Suite (BBraun Aesculap, Tuttlingen, Germany) is an image free kinematic navigation system used for hip navigation in orthopaedic surgery. According to the manufacturer's technical specifications, the accuracy of the system is $\pm 2 \mathrm{~mm}$ and $\pm 2^{\circ}$. However, the accuracy of this system remains to be determined independently of the manufacturer. Previous research has been undertaken using radiographs to compare its clinical accuracy against conventional hip replacements. The OrthoPilot based hip replacements showed more accurate positioning compared to the conventional hip replacements. But still with a range of positional errors [13 \& 14]. This clinical validation is important; however, it contains many possible sources of error or deviation from an ideal outcome in terms of the surgeons' use of the system, inaccurate palpation of landmarks, variation in actual cup position from that given by the navigation system and measurement of the final cup position. It is, therefore, not possible to validate the claims of the manufacturer regarding the accuracy of the system itself from these data. There is no literature evaluating the technical accuracy of the software, i.e. the accuracy of the system with the 
given known inputs. The main aims of this study were, therefore, to investigate the accuracy of OrthoPilot data while identifying the anterior pelvic plane (APP) of a phantom and to validate the navigation algorithm inside this CT free system (OrthoPilot system) which determines the position of the native acetabulum and hence the implanted cup. OrthoPilot validation was performed and compared against the gold standard of a VICON motion analysis system (Oxford metrics Ltd, Oxford, UK).

\section{Materials and Methods}

The system assessed was OrthoPilot ${ }^{\mathrm{TM}}$ navigation system with a Spectra camera from Northern Digital Inc. (Ontario, Canada). The software used was Hip Suite THA cup only navigation software Version 3.1. The validation was performed and compared against VICON Nexus version 1.4.116 with Bodybuilder software version 3.55 (Oxford metrics Ltd, Oxford, UK).

Experimental Set up: The experimental set up is shown in Figure 1. Both systems were under the same experimental conditions and capable of capturing the 3D location of the passive tracker marker clusters. The author was very careful in arranging both systems without interfering with each other, especially the VICON cameras. The pelvic phantom was positioned on a table in a supine position. In addition, the reference tracker was attached to a Goniometer and placed on the operated side of the pelvic phantom.

Concurrent validity between OrthoPilot and VICON was measured with a calibrated pelvic phantom model (Figure 2a). This phantom model was made to imitate the average size of the human pelvis with $14^{\circ}$ of anteversion and $45^{\circ}$ of anteversion (as determined by local analysis of pelvic CTs). These angle values were verified by engineering measuring techniques after the block had been machined. The physical dimensions of the phantom are explained as follows. The average distance between left and right anterior superior iliac supine was $230 \mathrm{~mm}$. The distance between the mid point of anterior superior iliac spines and mid point of the pubic Symphysis was $90 \mathrm{~mm}$. This phantom was machined with a grid of "peg" points around the exact landmark points of RASIS (Right Anterior Superior Iliac Spine), LASIS (Left Anterior Superior Iliac Spine) and PS (Pubic Symphysis). The grid of peg points allowed the addition of known deviation from the true landmarks position in caudal/cranial and medial/lateral directions.

This study was carried out with the passive tracker instrument set consisting of two rigid body clusters, each having 4 retro-reflective spheres on them in a unique arrangement that enabled the tracking and identification of each rigid body (tracker). One tracker was used as a reference and the other attached to a pointer that was used to identify points in space. Nominal patient details, position during surgery, surgical approach and implant type were input at the beginning of OrthoPilot navigation process to initiate the software. All anatomical landmark data were captured by keeping the five conditions below unchanged throughout the experiment; Patient's sex - female, Patient's position during the surgery - supine, Surgical approach - right hand side anterior approach, Implant cup type - plasma cup, Diameter of the trial cup - 48 $\mathrm{mm}$. 
Anatomical landmarks of right anterior superior iliac spine, left anterior superior iliac spine and Pubic Symphysis were palpated to define the APP by following OrthoPilot surgical navigation procedure. VICON data were captured for the same anatomical landmarks simultaneously. Data were captured by 12 VICON cameras and they were exported to static trial modeling. Static trial modeling was performed according to the program run by BodyBuilder software. After executing the program, position coordinates of each landmark were stored. Distance data between the anatomical landmarks were captured simultaneously from both OrthoPilot and VICON systems for the "supine" position of the phantom (APP horizontal). Each landmark bed was machined with several palpation points as seen in the Figure 2a. This allowed the definition of different widths and heights of pelvis within the same phantom model. In addition, above-mentioned palpation procedures were followed for three different sizes of APPs. They were defined as APP1, APP2 and APP3 according to width and height of the pelvis. They are shown in Figure 2b. RASIS-LASIS-PS landmarks were palpated 100 times for each APPs.

Distances between landmarks on the phantom were compared to the data recorded by the OrthoPilot system. Position vectors of each anatomical landmark from OrthoPilot system were extracted from relevant transformation matrices, while position vectors from VICON system were extracted from the data using BodyBuilder. The BodyBuilder program contained calculations of position coordinates of the landmarks. Once executed it, calculated landmark coordinates in global frame and these were stored in ASCII file format. Then, those coordinates were exported to Excel for the final calculations. For a single APP measurement each anatomical landmark was palpated once.

The cup navigation algorithm accuracy was tested by unpicking OrthoPilot algorithm and applying a similar algorithm to calculate the native anteversion and inclination angles of the acetabulum using the VICON system. In the OrthoPilot surgical navigation procedure, the first step was to palpate the anatomical landmarks of APP (RASIS, LASIS and PS) and then, system records the deepest point of the acetabular (For that, centre point of the grid of acetabular plane of the phantom was palpated as shown in the Figure 2a). The next step was the trial cup registration where system determines the acetabular cup axis. With this acetabular cup axis data and the APP data, acetabular angles are calculated for the native acetabulum. Radiographic definition of the anteversion and inclination angles were calculated according to the Murray [15]. The angle calculation algorithm is shown in Figure 3. Initially, orientation of the native acetabulum was obtained on multiple occasions without varying the APP, by using APP2 shown in Figure $2 b$ (the mid size APP).

Subsequently, the APP was changed by varying the anatomical landmarks in coronal plane using the grid of error points. One anatomical landmark was changed at a time while keeping the other two landmarks the same. To do this the surgical tool was moved in the Caudal/Cranial and Medial/Lateral directions to the adjacent machined hole (Figure 4). There was no vertical movement (anterior-posterior) of the tool. First, the RASIS was moved from its original position along the lateral direction by $10 \mathrm{~mm}$ while leaving LASIS and PS stationary. At the next stage, RASIS was moved by $20 \mathrm{~mm}$ in the lateral direction and then by 10 and $20 \mathrm{~mm}$ in medial direction. Subsequently, the same procedure was followed for the RASIS in 
the medial and lateral directions. Then, caudal and cranial displacements of $10 \mathrm{~mm}$ and $20 \mathrm{~mm}$ were applied again while keeping LASIS and PS stationary. This procedure was repeated for LASIS and PS while again keeping the other two landmarks stationary.

\section{Results}

The distances between landmarks from both OrthoPilot and VICON systems were compared with the calibrated distances from the phantom model. Mean value of the distances between pairs of anatomical landmarks and their standard deviations are shown in Table 1. It can be seen that mean value of the distance between landmarks were almost identical between systems and when compared to the phantom. The standard deviations are less than $1 \%$ of the measured value and less than $1 \mathrm{~mm}$ in all cases.

Comparison was also made for anteversion and inclination angles of the acetabulum of the pelvic model. Measurements of anteversion and inclination angles produced by both systems are very similar to the calibrated values (Table 2). More importantly the standard deviations (SD) of the angle values are less than $1 \%$ of the phantom values.

When the landmark position of the RASIS varied along the caudal and cranial directions by $10 \mathrm{~mm}$ and $20 \mathrm{~mm}$ (Figure 5a), the inclination angle deviated considerably $\left( \pm 5^{0}\right)$ compare to the inclination angle value of the pelvic phantom $\left(45^{\circ}\right)$. But the anteversion angle remained unchanged. Moving the RASIS in the medial or lateral direction left both inclination and anteversion angles unchanged.

When the LASIS was moved in caudal/cranial directions again the inclination angle changed considerably $\left( \pm 5^{0}\right)$ from its original value $\left(45^{0}\right)$, but with no deviation in anteversion angle (Figure 6a). These changes were the same magnitude as those seen in the RASIS movement except the changes were the mirror image of those for the RASIS. Again, no significant changes occurred in anteversion or inclination angles when the LASIS moved along medial and lateral axes as seen in Figure 6b.

When the landmark position of PS changes along caudal and cranial axis (Figure 7a) or medial and lateral axis (Figure 7b), only small changes in inclination and anteversion angle were observed.

The inclination angle deviation observed, due to landmark changes at LASIS and RASIS along the caudal/cranial direction can be explained with reference to the Figure 8. If the APP changes with a caudal displacement $(p)$ of $10 \mathrm{~mm}$, then the angular error $\left(\theta_{\text {ERROR }}\right)$, then $\theta_{\text {ERROR }}=\tan ^{-1}(p / t)$. When $\mathrm{p}=$ $10 \mathrm{~mm}$ and $\mathrm{t}=230 \mathrm{~mm}$ gives $\theta_{\text {ERROR }}=3^{0}$. This means, inclination angle changes by $\pm 3^{0}$ when either LASIS or RASIS position changes by $10 \mathrm{~mm}$ in caudal $/$ cranial axis. Similarly, when $\mathrm{p}=20 \mathrm{~mm} \theta_{\text {ERROR }}=$ $5^{0}$ and inclination angle changes by $\pm 5^{0}$. 
If the size of the APP small is (APP1) then $\mathrm{t}=170 \mathrm{~mm}$ and $\mathrm{p}=10 \mathrm{~mm}$ and $20 \mathrm{~mm}$, then $\theta_{\text {ERROR }}=4^{0}$ and $7^{0}$ respectively. For the large size APP $(\mathrm{APP} 3), \mathrm{t}=290 \mathrm{~mm}$, and $\mathrm{p}=10 \mathrm{~mm}$ and $20 \mathrm{~mm}$, then $\theta_{\text {ERROR }}=2^{0}$ and $4^{0}$, respectively. Therefore, in summary caudal/cranial displacement of the position of the RASIS or LASIS leads to an error in the inclination angle which increases with increasing positional error and with decreasing pelvic size.

\section{Discussion}

This research study was conducted to validate the accuracy of a CT free navigation system (OrthoPilot hip navigation) process from a measurement point of view. According to the manufacturer's technical specifications, the accuracy of the system is defined as $\pm 2 \mathrm{~mm}$ and $\pm 2^{0}$. The accuracy of this system required to be determined independently of the manufacturer. Clinical validation contains many sources of error or deviation from an ideal outcome in terms of surgeons' use of the system. These errors include inaccurate landmark palpation, variation in actual cup position (that given by the navigation system) and measurement of the final cup position.

A previous study has concluded that the accuracy of acetabular component positioning using free hand, CT-based and imageless navigation as follows: with conventional method 53\% of the components were outside the Lewinneck's safe zone [16] and 7\% when using imageless navigation [17]. These results show the accuracy and repeatability of image-free navigation such as the OrthoPilot system. OrthoPilot accuracy and repeatability were observed during this study. Results for the distance between the anatomical landmarks were within the range of $\pm 2 \mathrm{~mm}$ to the exact distance reading. In addition, results for the acetabular angle were within the range of $\pm 2^{0}$ to the exact angle values with small standard deviations. Small standard deviations show the precision of the OrthoPilot results.

However, errors introduced during landmark palpation have a substantial effect on the final cup orientation and hence potentially on impingement, dislocation, wear and loosening [18]. Lee et al., discussed the acetabular angle errors introduced due to the fat tissue thickness [19]. Fat tissue thickness introduces coordinate errors mainly in the anterior axis. However, except for the fat tissue thickness, errors can be introduced with the incorrect landmark registration due to the deviation of the exact point in the coronal plane. These factors result to position the implant incorrectly. The above fact can be clearly seen from the resultant acetabular angles in this study, when varying the anterior pelvic plane in coronal plane.

A deviation of landmark location was shown to lead significant errors in the anatomical reference particularly the inclination angle when displaces in the caudal/ cranial direction. Therefore, the accuracy of point registration plays an important role during surgery. A $20 \mathrm{~mm}$ deviation of the exact landmark position of RASIS or LASIS along caudal/cranial direction can lead to change the inclination angle by $5^{0}$ for an average sized pelvis. This inclination angle error will vary with the size of the pelvis. 
Data for the distance between anatomical landmarks were within the range of $\pm 2 \mathrm{~mm}$ to the exact distance reading. All the distance results were observed to have small standard deviations. Small standard deviations represent the precision of OrthoPilot results. According to OrthoPilot manufacture's technical specification distance accuracy is $\pm 2 \mathrm{~mm}$ and this was verified with the distance data recorded. The VICON with its multiple cameras gave more accurate and precise results than the OrthoPilot with its two cameras. The use of multiple camera systems could further improve the accuracy and precision of such system and would improve line of sight issues. Therefore, it can be stated herein that OrthoPilot instrument position data are accurate enough for current surgical operations.

Data of the acetabular angles were within the range of $\pm 1^{0}$ to the exact acetabular angle readings of the pelvic phantom. It provided that there was caudal/cranial misplacement of the RASIS with respect to the LASIS of less than $10 \mathrm{~mm}$. All these results were observed with small standard deviations. According to OrthoPilot manufacture's technical specifications angular accuracy is $\pm 2^{0}$ and that is verified by the experimental results. Therefore, it can be stated herein that the Cup navigation algorithm produces accurate angle results provided the landmarks are correctly identified.

Changing the landmark positions of RASIS and LASIS along caudal/cranial axis reduces the accuracy of APP identification resulting in deviations of the inclination angle larger than the manufacture's estimates $\left(> \pm 2^{0}\right)$. However, errors in the landmark position along medial-lateral axis do not affect either anteversion or inclination angles to any measurable degree. When similar changes occur at the landmark position of PS, they do not change anteversion or inclination angles. Therefore, care should be taken when registering the RASIS and LASIS not to displace them in the caudal/cranial directions.

All the experiments were conducted with a calibrated pelvic phantom model. OrthoPilot data were compared against the gold standards of the VICON system. All the OrthoPilot data produce comparable results to the VICON data and calibrated pelvic phantom model data. This shows that OrthoPilot data capturing process is highly accurate and repeatable in engineering point of view.

During this study, radiographic definition of the acetabular orientation was examined. However, OrthoPilot is used to measure acetabular angles for anatomical and operative definitions. Same experimental procedure used above can be applied to check accuracy of the acetabular orientation for anatomical and operative definitions. In this situation, angle calculation algorithm used for VICON data should be changed according to the anatomical and operative definitions. Angles should only be measured for the neutral position of the APP. It is expected to observe similar acetabular angle deviations due to the APP variation as observed for the radiographic definition of the angle calculation. This study was carried out for the cup only navigation. Similar procedure can be applied to validate the OrthoPilot based femoral navigation process in engineering point of view. For that, modification should be introduced to the pelvic phantom which was used during this study. In addition, calibrated femoral phantom should be made to attach to the pelvic phantom. Experiments can be organized to measure some femoral parameters like leg length and femoral diameter. 


\section{Conclusions}

The data obtained from OrthoPilot are comparable to these obtained from the gold standard VICON system and the calibrated distances of the phantom. Small standard deviations of less than $1 \%$ of actual value illustrate the precision of data capturing. We conclude that, OrthoPilot data capturing process is accurate when applied to a metal phantom. Acetabular angles obtained from OrthoPilot were equivalent to these obtained from VICON and the calibrated phantom angles, when APP was exactly on the RASIS, LASIS and PS. Smaller standard deviations of less than $1 \%$ of actual angle values were obtained for the acetabulum of the pelvic phantom. These findings conclude that, OrthoPilot cup navigation algorithm produces accurate results when care is taken with landmark registration, particularly registering the RASIS and LASIS in the caudal/cranial direction.

\section{References}

1. Erhardt K, Report of joint meeting of the sections of Geriatrics \& Gerentology and Rheumatology \& Rehabilitation. Osteoarthritis in old age. J. R. Soc. Med. 1995; 88: 539 - 542.

2. Dandy D. J and Edwards D. J., (2003), Essential Orthopaedics and Trauma. 3rd ed. Churchill Livingston, Oxford, UK. ISBN 0443072132

3. Frankel S, Eachus J, Pearson N, Greenwood R, Chan P, Peters TJ, et. al Population requirements for primary hip replacement surgery: a cross sectional study. The Lancet 1999; 353(9161): 1304 $-1309$.

4. DiGioia III A.M, , Jaramaz B, Plakseychuk A. Y, Moody J. E, Nikou C, LaBarca R. S, Levison T. J, and Picard F, (2002), Comparison of a Mechanical Acetabular Alignment Guide With Computer Placement of the Socket. The Journal of Arthroplasty, Vol. 17 No. 3

5. Numair J, Joshi A. B, Murphy J. C, Porter M. L, Hardinge K, (1999), Total hip arthroplasty for congenital dysplasia or dislocation of the hip, Survivorship analysis and long-term results. J Bone Joint Surg Am, 79:1352-1360

6. Fender D, Harper W. M, Gregg P. J, (1999), Outcome of Charnley total hip replacement across a single health region in England, Journal of bone and joint surgery, 81-B(4): 577 - 581

7. Herberts $P$ and Malchau H, (2000), Long-term registration has improved the quality of hip replacement. A review of the Swedish Total Hip Replacement registers comparing 160,000 cases. Acta. Orthop. Scan. 71(2): 111 - 121

8. Kennedy J.G, Rogers W.B, Soffe K.E, Sullivan R.J, Griffen D.G, Sheehan L.J. (1998), Effect of acetabular component orientation on recurrent dislocation, pelvic osteolysis, polyethylene wear, and component migration. Journal of Arthroplasty. 13:530-4

9. Sugano N, Computer-assisted orthopaedic surgery, Journal of Orthopaedic Science, The Japanese Orthopaedic association 2003; 8:442-448

10. Tran HH, Matsumiya K, Masamune K, Sakuma I, Dohi1 T , Liao H, Interactive 3D Navigation System for Image-guided Surgery, The International Journal of Virtual Reality, 2009; 8(1): 9-16 
11. Kelley TC, Swank M L, Role of Navigation in Total Hip Arthroplasty, Journal of Bone \& Joint surgery 2009; volume 91-A

12. DiGioia III AM, Jaramaz B, Plakseychuk AY, Moody JE, Nikou C, LaBarca RS, et al, Comparison of a mechanical acetabular alignment guide with computer placement of the socket. The Journal of Arthroplasty 2003; Vol. 17

13. Kalteis T, Handel M, Bäthis H, Perlick L, Tingart M, Grifka J, Imageless navigation for insertion of the acetabular component in total hip arthroplasty, Journal of Bone Joint Surgery [Br]2006;88-B:163-7

14. Kiefer $\mathrm{H}$, OrthoPilot cup navigation - How to optimize cup positioning, International Orthopaedics (SICOT) , 2003 - 27 (Suppl.1):S37-S42

15. Murray DW, The Definition and measurement of acetabular orientation. The Journal of Bone and Joint Surgery 1993; 75-B: 228-32.

16. Lewinnek G.E, Lewis J.L, Tarr R, Compere C.L, Zimmerman J.R, (1978), Dislocations after total hip-replacement arthroplasties. J Bone Joint Surg Am 60:217-220.

17. Kalteis T, Handel M, Bäthis H, Perlick L, Tingart M, Grifka J, (2006), Imageless navigation for insertion of the acetabular component in total hip arthroplasty, J Bone Joint Surg Brit Med;88B:163-167

18. Wolf A, DiGioia III AM, Mor AB, Jaramaz B, Cup alignment error model for THA, Clinical orthopaedic and related research, 2005 - 437, pp 132-137.

19. Lee YS and Yoon TR, Error in acetabular socket alignment due to the thick anterior pelvic soft tissues, The Journal of Arthroplasty, 2008, Vol. 23 No. 5, pp 699-705

20. Świątek-Najwer E, Będziński R, Krowicki P, Krysztoforski K, Keppler P, Kozak J, Improving surgical precision-application of navigation system in orthopaedic surgery. Acta of Bioengineering and Biomechanics 2008; Vol. 10, No. 4.

21. Clarke J.V, Deakin A.H, Nicol A.C, Picard F, Measuring the positional accuracy of computer assisted surgical tracking systems, Computer Aided Surgery, 2010, 15(1-3):13-18,

22. Liaw CK, Yang RS, Hou SM, Wu TY, Fuh CS, A simple mathematical standardized measurement of acetabulum anteversion after total hip arthroplasty. Computational and Mathematical Methods in Medicine June 2008; Vol. 9, No. 2, 105-119. 


\section{Biographical notes}

Shanika MA Arachchi was an MPhil student at Department of Biomedical Engineering, University of Strathclyde, Glasgow UK.

Angelica S. Augustine was an MPhil student at Department of Biomedical Engineering, University of Strathclyde, Glasgow and a researcher at Golden Jubilee National Hospital, Clyde Bank, UK

Angela H Deakin is an honorary research fellow, Department of Biomedical Engineering, University of Strathclyde, Glasgow, and clinical research coordinate at Department of Orthopaedics, Golden Jubilee National Hospital, Clyde Bank, UK

Frédéric Picard is a visiting Professor at Department of Biomedical Engineering, University of Strathclyde, and an Orthopaedic surgeon at Department of Orthopaedics, Golden Jubilee National Hospital, Clyde Bank, UK

Philp J Rowe is a Professor of rehabilitation science at Department of Biomedical Engineering, University of Strathclyde, Glasgow, UK 


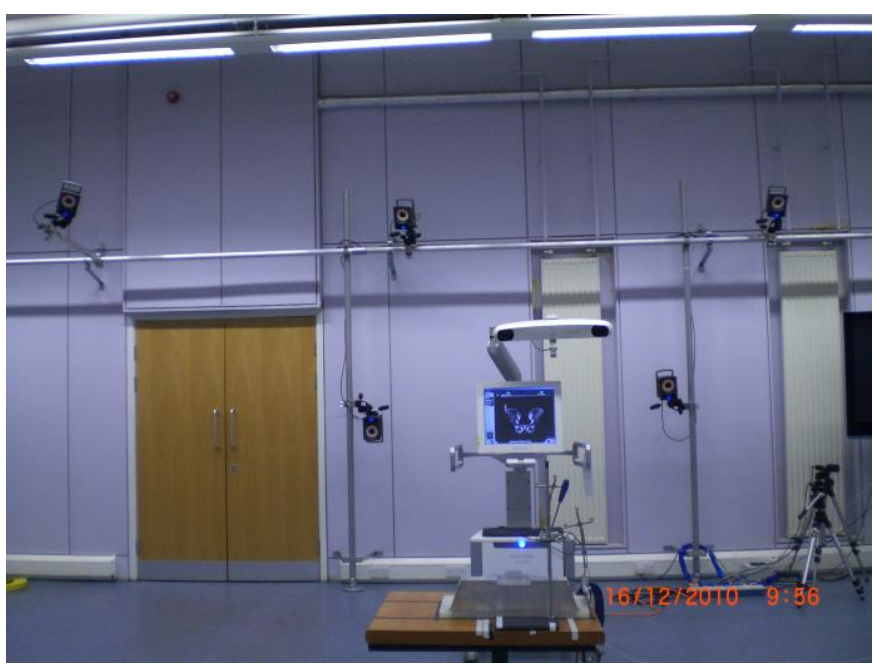

Figure 1. Simultaneous data recording from OrthoPilot and VICON systems

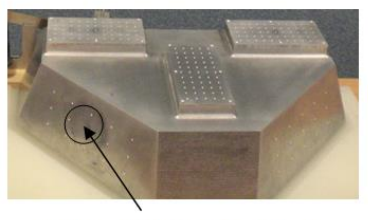

Point to palpate the deepest point of the acetabular (a)

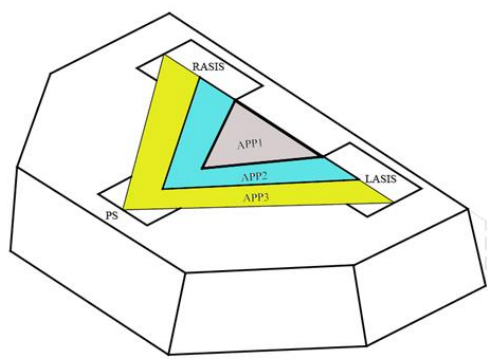

(b)

APP1 : RASIS-LASIS 170 mm, RASIS-PS 102 mm, LASIS- PS 102 mm APP2 : RASIS-LASIS $230 \mathrm{~mm}$, RASIS-PS $145 \mathrm{~mm}$, LASIS- PS $145 \mathrm{~mm}$ APP3 : RASIS-LASIS 290 mm, RASIS-PS 190 mm, LASIS- PS 190 mm

Figure 2a Pelvic phantom model - 2b Anterior pelvic planes on phantom 


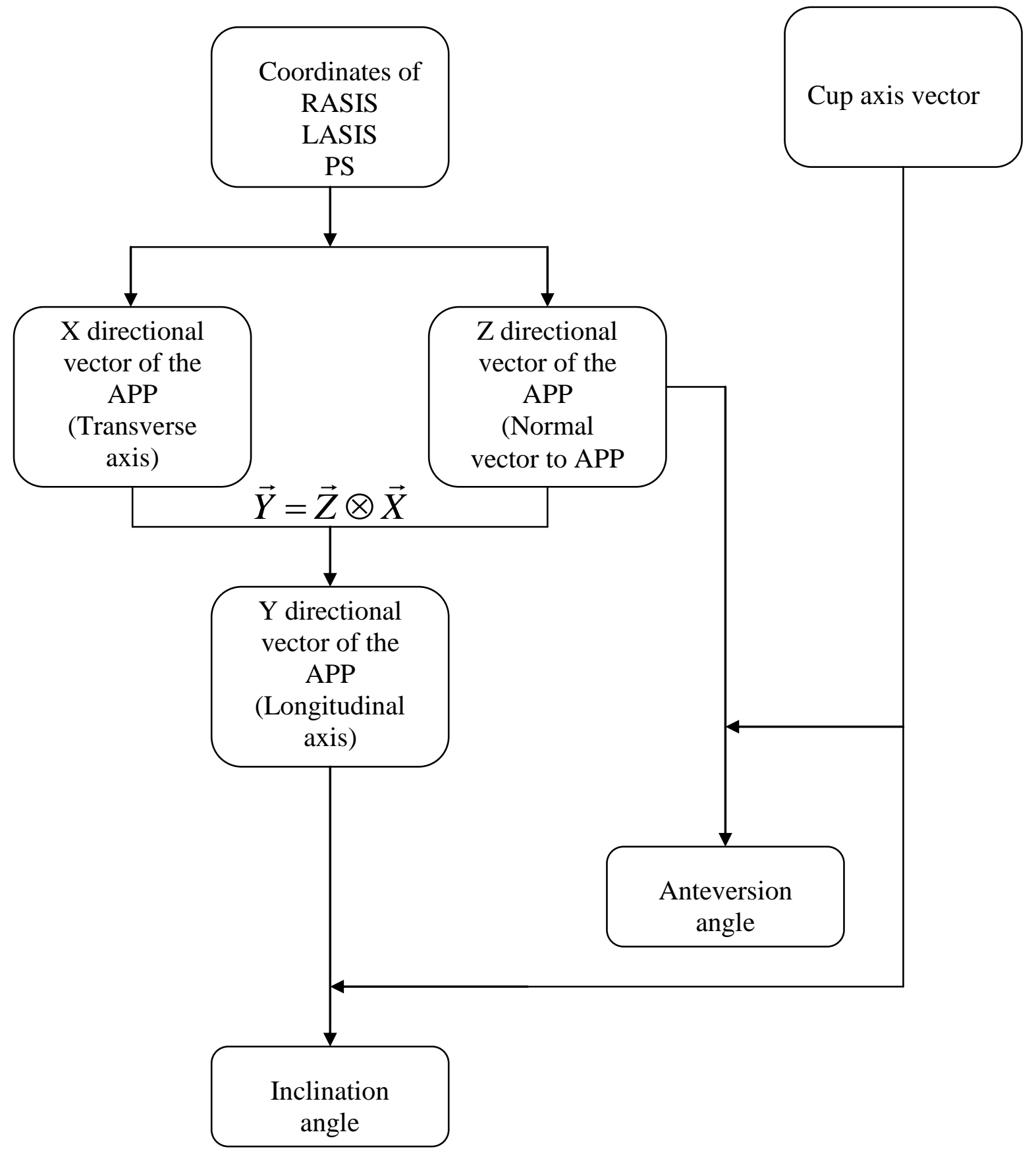

Figure 3. Flow chart of angle calculation algorithm 


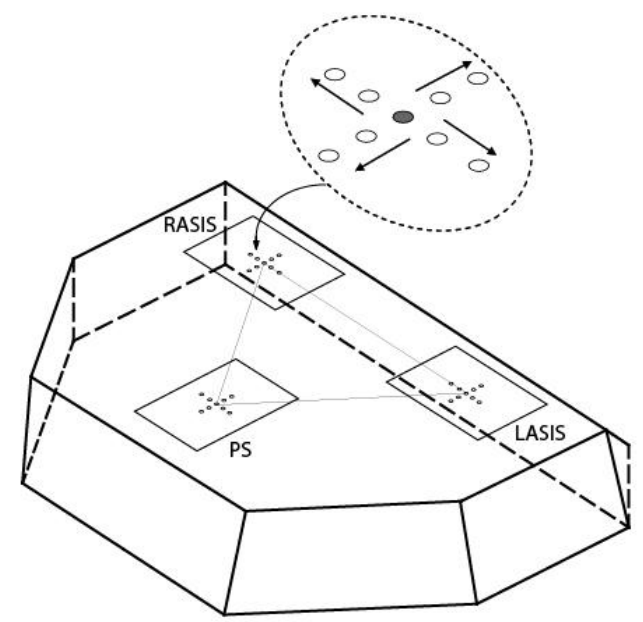

Figure 4. Landmark variations

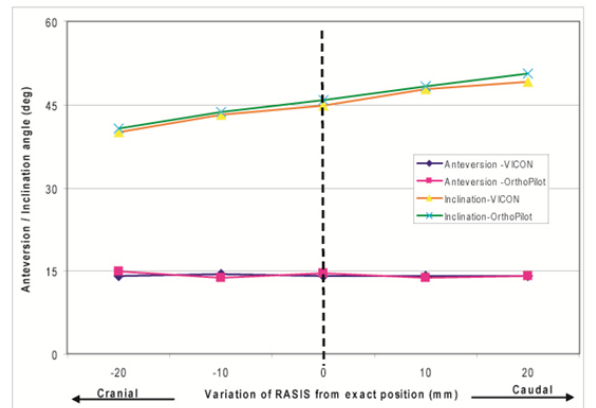

(a)

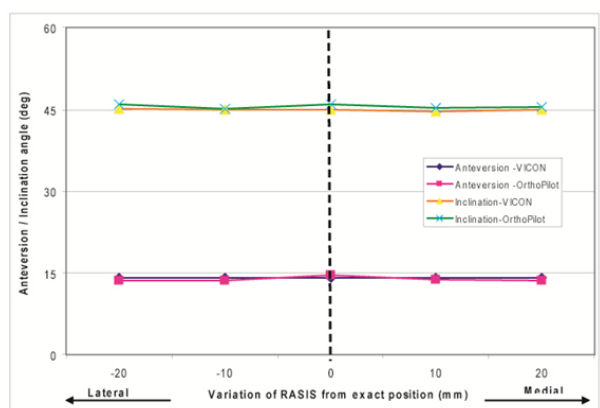

(b)

5a Along Caudal and Cranial directions

5b Along Medial and Lateral directions

Figure 5. Graphical representation of the acetabular angle variation when RASIS changes

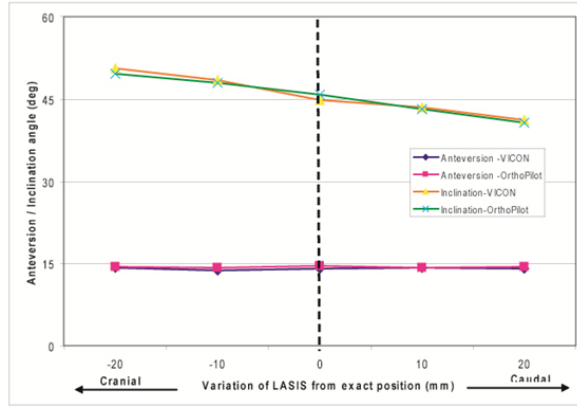

(a)

6a Along Caudal and Cranial directions

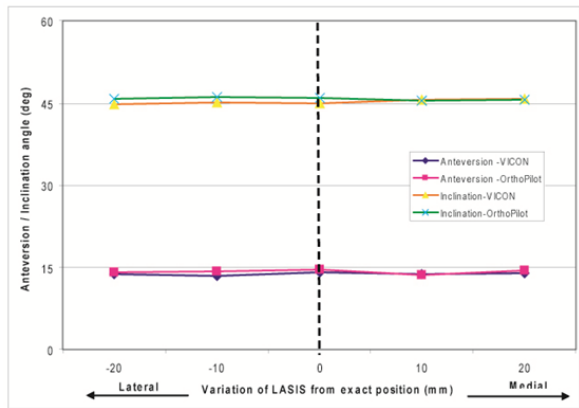

(b)

Figure 6. Graphical representation of the acetabular angle variation when LASIS changes 


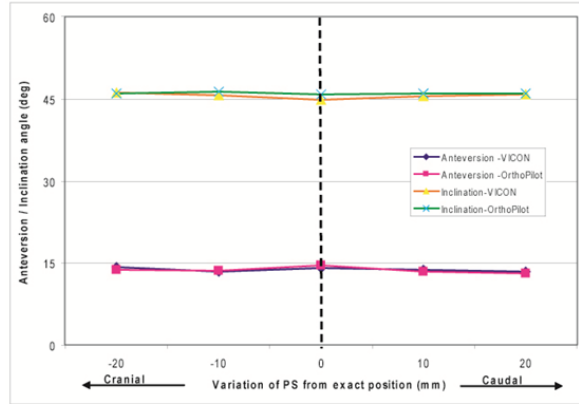

(a)

7a Along Caudal and Cranial directions

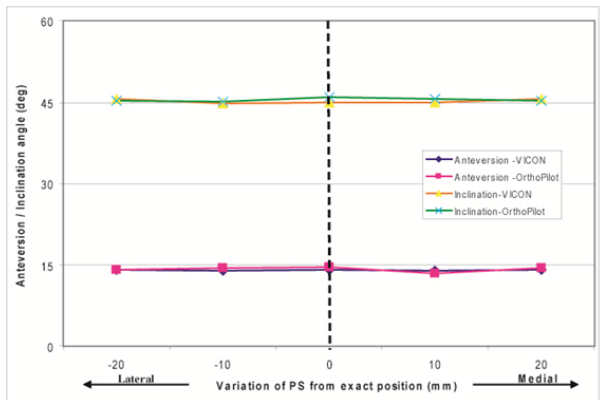

(b)

7b Along Medial and Lateral directions

Figure 7. Graphical representation of the acetabular angle variation when PS changes

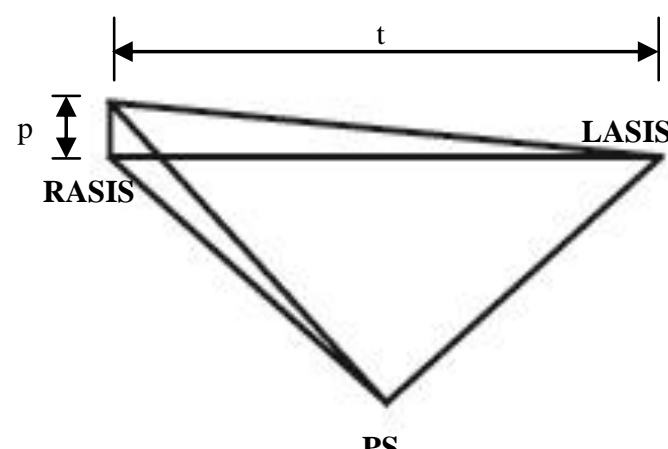

PS
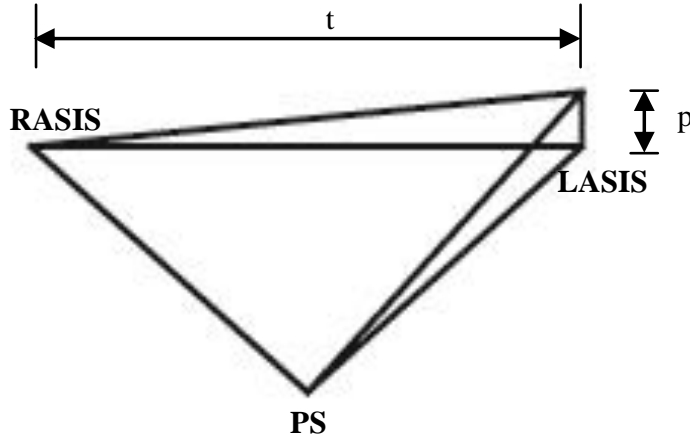

8a when changing the landmark of RASIS along caudal and cranial direction

$\mathbf{8 b}$ when changing the landmark of LASIS along caudal and cranial direction

Figure 8. APP variations 
Table 1. Distance comparison between anatomical landmarks of RASIS-LASIS, RASIS-PS and LASISPS.

\begin{tabular}{|c|c|c|c|c|c|c|}
\hline \multirow[t]{2}{*}{ APP types } & \multirow{2}{*}{$\begin{array}{l}\text { Distance between } \\
\text { anatomical } \\
\text { landmarks }\end{array}$} & \multirow{2}{*}{$\begin{array}{l}\text { Phantom model } \\
\text { data }(\mathrm{mm})\end{array}$} & \multicolumn{2}{|c|}{ VICON } & \multicolumn{2}{|c|}{ OrthoPilot } \\
\hline & & & $\begin{array}{l}\text { Mean } \\
\text { Value } \\
(\mathrm{mm}) \\
\mathrm{n}=100\end{array}$ & $\mathrm{SD}$ & $\begin{array}{l}\text { Mean } \\
\text { Value } \\
(\mathrm{mm}) \\
\mathrm{n}=100\end{array}$ & $\mathrm{SD}$ \\
\hline \multirow{3}{*}{ APP 1} & RASIS-LASIS & 170 & 171 & 0.08 & 170 & 0.15 \\
\hline & RASIS-PS & 102 & 102 & 0.09 & 104 & 0.14 \\
\hline & LASIS-PS & 102 & 102 & 0.10 & 104 & 0.17 \\
\hline \multirow{3}{*}{ APP 2} & RASIS-LASIS & 230 & 231 & 0.11 & 230 & 0.19 \\
\hline & RASIS-PS & 145 & 144 & 0.17 & 146 & 0.19 \\
\hline & LASIS-PS & 145 & 145 & 0.09 & 146 & 0.34 \\
\hline \multirow{3}{*}{ APP3 } & RASIS-LASIS & 290 & 290 & 0.09 & 290 & 0.42 \\
\hline & RASIS-PS & 190 & 190 & 0.14 & 189 & 0.98 \\
\hline & LASIS-PS & 190 & 189 & 0.21 & 189 & 0.54 \\
\hline
\end{tabular}

Table 2. Acetabular angle comparison.

\begin{tabular}{|c|c|c|c|c|c|}
\hline \multirow[b]{2}{*}{ Angle } & \multirow{2}{*}{$\begin{array}{l}\text { Phantom model } \\
\text { data } \\
\text { Value (deg) }\end{array}$} & \multicolumn{2}{|l|}{ VICON system } & \multicolumn{2}{|l|}{ OrthoPilot system } \\
\hline & & $\begin{array}{l}\text { Mean Value } \\
(\mathrm{deg}) \\
\mathrm{n}=100\end{array}$ & SD & $\begin{array}{l}\text { Mean Value (deg) } \\
n=100\end{array}$ & SD \\
\hline Anteversion angle & 14 & 14.07 & 0.10 & 14.54 & 0.13 \\
\hline Inclination Angle & 45 & 44.87 & 0.07 & 45.86 & 0.15 \\
\hline
\end{tabular}

